\title{
Synchronising horizontal arm movement with transparent motion
}

\author{
Lizzy Bleumers $^{\mathrm{a}, *}$, Tanja Ceux ${ }^{\mathrm{b}}$, Martinus Buekers ${ }^{\mathrm{b}}$, Johan Wagemans ${ }^{\mathrm{a}}$

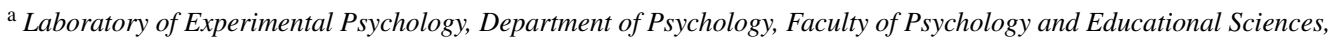 \\ K.U. Leuven, Tiensestraat 102, B-3000 Leuven, Belgium \\ ${ }^{\mathrm{b}}$ Perception and Performance Lab, Department of Biomedical Kinesiology, Faculty of Kinesiology and Rehabilitation Sciences, \\ K.U. Leuven, Tervuursevest 101, B-3001 Heverlee, Belgium \\ Received 12 June 2006; accepted 19 June 2006 \\ Available online 25 July 2006
}

\begin{abstract}
The relationship between perception and motor performance was studied in a situation that required perceptual processing of a complex motion stimulus in which a target signal had to be segmented, selected, and tracked. Participants were asked to move their arm in synchrony with one surface of a transparent motion display in which two surfaces moved horizontally back-and-forth over each other. The quality of tracking performance was measured as a function of bottom-up and top-down perceptual cues and their interplay. Target signal strength was manipulated by lowering the relative amount of signal dots constituting the target, i.e., the coherence level $(100 \%-50 \%-30 \%-10 \%$; the distractor surface was always $100 \%$ coherent). A colour cue that distinguished the target from the distractor surface was either available or absent. In the presence of a colour cue, participants experienced little or no difficulties at coherence levels of 50\%-100\% but when surface formation was complicated by lowering the coherence level, synchronisation consistency decreased. This corresponds with continuous attempts, successful and unsuccessful, to correct inaccurate synchronisation. In the absence of a colour cue, difficulties were frequently observed in all coherence conditions, but they differed depending on the coherence level. Overall, these results suggest that colour can serve as a strong top-down cue for proper target selection and tracking, provided that bottom-up motion signals are sufficiently strong.
\end{abstract}

(C) 2006 Elsevier B.V. All rights reserved.

Keywords: Synchronisation; Transparent motion; Random-dot kinematogram; Surface formation; Tracking; Perception-action coupling

\section{Introduction}

Our everyday behaviour requires a precise interplay between what we see and how we act. Navigating through traffic, doing sports, cooking are just a few examples of daily actions that necessitate us to accurately perceive our environment and adapt our behaviour accordingly. One crucial aspect of perception in this respect is picking up motion, through segregation and integration of local motion signals. Elements with a common motion direction and speed tend to be grouped together according to the Gestalt principle of common fate $[14,17]$. These grouping processes are typically studied by means of the motion coherence paradigm [12]. In this paradigm random-dot kinematograms are used, in which a number of dots, the signal dots, move coherently while the other dots, called noise dots, move randomly. Motion

\footnotetext{
* Corresponding author. Tel.: +32 16326075; fax: +32 16325924.

E-mail address: Lizzy.Bleumers@psy.kuleuven.be (L. Bleumers).
}

discrimination performance can then be quantified in terms of the required proportion of signal dots, i.e., the coherence level $[3,8]$ (see [16] for an alternative approach).

Ceux et al. [6,7] integrated the motion coherence paradigm in a synchronisation task to investigate motor performance coupled to motion perception. They examined how varying the coherence level of a stimulus (100\%-50\%-30\%-10\%) affected synchronisation behaviour. In their perceptuo-motor task participants had to continuously match the movement of their right arm in space and time with the horizontal cyclical motion of coherently moving dots. Synchronisation accuracy and consistency were considerably reduced when only $10 \%$ of the displayed dots moved coherently [6].

In the current study, we extend the research of Ceux et al. to transparent motion stimuli. Transparent motion entails two stimulus components moving transparently across each other so that two different motions have to be assigned to the same retinal position [13], which increases the contribution of segregation and integration of local motion signals. Specifically, we 
examined synchronisation behaviour at different coherence levels, analogous to the study of Ceux et al. [6], when a fixed amount of dots was also present moving coherently in the opposite direction of the synchronisation target. In the previous synchronisation studies [6,7] the visual system had to select a group of coherently moving dots embedded in a background of randomly moving dots, whereas in the present paradigm an extra surface was added which competes for selection (i.e., the distractor surface). Given the results for the original stimuli, we expected synchronisation problems to become apparent at low coherence levels. We anticipated that participants would start to incorrectly track the stable distractor surface under these conditions.

We wanted to investigate whether synchronisation to the target could be improved by giving the distractor surface a different colour. Such a colour cue could aid selection of the synchronisation target during the trial, maintaining focus on it and ignoring the salient distractor surface by defining the relevant object for the task. Convincing evidence supports the idea that objects can serve as units for attention (e.g. [1], see [11] for a review). Furthermore, transparent motion stimuli are a useful test case for isolating object-based attention from space-based attention because the components competing for selection overlap [15]. Valdes-Sosa et al. [15] found advantages in transparent motion stimuli for focussed attention on one surface over attention divided between two differently coloured surfaces when participants made perceptual judgments. This suggests that colour can be used to select surfaces and objects attentively. Based on this evidence, we predicted that participants would be better at selecting the target and synchronising their arm movement with it when a colour cue was present.

How the coherence and colour manipulation would interact, was an open question. Colour can serve as a strong top-down cue for target selection, but proper selection and tracking require sufficiently strong bottom-up motion signals. At low coherence levels, the target surface percept might be unstable or even absent due to unsuccessful segmentation of the display. This would reduce or perhaps even eliminate beneficial effects of colour.

\section{Method}

\subsection{Participants}

Sixteen students (mean age $=22.31$ years, age range $19-28$ ) volunteered to participate in the study. The participants had normal or corrected to normal vision and had no previous experience with the task. The volunteers gave their informed consent prior to taking part in the experiment. A computer based screening test, a short online version of the Ishihara test [10], excluded red-green colour vision deficiencies among the participants.

\subsection{Apparatus and stimuli}

In a darkened room, the participants were seated behind a table with their right forearm positioned on a lever while grasping a handle fixed to the distal end. The lever could freely rotate in the horizontal plane and the participant's elbow was placed just above the lever's axis of rotation. A shaft encoder (Tamagawa TS5116) was connected to the axis of rotation of the lever and registered its position (accuracy of $0.1^{\circ}$ ) at a rate of $100 \mathrm{~Hz}$. The dynamic stimulus was a randomdot kinematogram, generated in MATLAB, using the Psychophysics Toolbox extensions [2,9]. It was displayed as a large black rectangle $(1.17 \mathrm{~m} \times 1.19 \mathrm{~m}$; $\left.43.94^{\circ} \times 44.62^{\circ}\right)$ continuously filled with the total number of 1000 dots, divided in two sets of dots each containing 500 dots. An individual dot was about $2 \mathrm{~cm}$ in size $\left(0.79^{\circ}\right)$. The first set of dots contained the synchronisation target. This set consisted of a percentage of signal dots moving sinusoidally back-and-forth, starting from the left to the right, in a coherent way at a rate of $0.3 \mathrm{~Hz}\left(26^{\circ} / \mathrm{s}\right)$, while the remaining dots (noise) moved randomly (each noise dot was assigned an arbitrary direction). In contrast, the other set of dots only contained coherently moving signal dots which gave rise to a salient distractor surface. These dots also moved horizontally back-and-forth, but always in the opposite direction of the synchronisation target. Between the two reversal positions, all dots had a limited lifetime of five frames $(0.080 \mathrm{~s})$ of the total sequence of 100 frames $(1.65 \mathrm{~s})$. This was done to avoid the possibility of tracking one dot or a small group of dots. The visual signal was projected on a wall in front of the participants at a viewing distance of $1.45 \mathrm{~m}$ by means of a LCD-projector with a spatial resolution of $800 \times 600$ pixels $($ NEC MultiSync VT 540).

\subsection{Procedure}

Before starting the actual experiment, participants were given a short test to preclude problems with the task due to red-green colour vision deficiencies. Participants were then instructed on the synchronisation task. They were asked to move their right forearm so that their motion corresponded with the horizontal trajectory of the target (peak-to-peak amplitude: $44^{\circ}$ ). After instructions, we showed examples of the stimuli to acquaint participants with the nature of the stimulus. Finally, the actual experiment began in which participants took part in all conditions. Participants had to complete 20 back-and-forth arm movement cycles per trial.

At the beginning of each trial a visual intro stimulus was shown, directly preceding the appearance of the transparent motion stimulus. Arm movement had to be initiated after the intro, when the transparent motion stimulus was displayed. During the intro 10 dots made one horizontal back-and-forth movement at the same frequency as the actual stimulus $(0.3 \mathrm{~Hz})$. The intro indicated what would be the synchronisation target, as the movement of the target was a continuation of the intro movement. Participants were free to move their eyes during the trial.

The stimulus had certain characteristics depending on the given condition. The coherence level of a group of 500 dots was manipulated $(100 \%-50 \%-30 \%-10 \%)$ which resulted in a more or less salient synchronisation target. Another group of 500 dots moved coherently and formed a stable surface, the distractor surface, which had to be ignored. There were two colour conditions. In the WW condition the dots of the distractor surface were not coloured differently from the other dots; all displayed dots were white. In the GR condition the distractor dots were red while all other dots (signal and noise dots) were green. For example, in the GR condition with $50 \%$ coherence a stimulus was displayed with green dots containing the synchronisation target and a red distractor surface. This stimulus contained 250 green coherently moving dots, 250 green noise dots and a distractor surface of 500 red dots moving coherently in the opposite direction of the target dots.

The administration of the experiment was organised in four blocks, one for each coherence level. The order of these blocks was varied across participants. Each block contained six trials: three successive trials of the WW condition, and three of the GR condition. Again, the order of these colour conditions within a block was varied across participants.

\subsection{Data description and analysis}

\subsubsection{Global parameters}

First, we measured the parameters typically used in synchronisation research [4-7]. These parameters capture performance on one trial in a single value. The mean amplitude $\left(^{\circ}\right)$ and mean within-participant variability of amplitude $\left(^{\circ}\right)$ were computed to study the spatial performance of the arm movement according to the criterion amplitude of the visual signal $\left(44^{\circ}\right)$. Further, the spatio-temporal characteristics of the synchronisation performance throughout the entire trajectory were described by the absolute mean (continuous) relative phase $\left(^{\circ}\right.$ ) and the mean within-participant variability of the (continuous) relative phase $\left({ }^{\circ}\right)$. The spatio-temporal parameters were obtained by calculating the absolute mean and the standard deviation of the relative phase values for the samples taken during a trial. These values give the difference in phase angle between 
Table 1

Selection criteria for trial classification

\begin{tabular}{|c|c|}
\hline Trial category & Selection criteria \\
\hline Target & $\begin{array}{l}13 \text { or more cycles with an absolute mean relative phase of } \\
\text { less than } 30^{\circ}\end{array}$ \\
\hline TargetDeviant & $\begin{array}{l}\text { Less than } 13 \text { cycles with an absolute mean relative phase of } \\
\text { less than } 30^{\circ} \\
\text { Four or more consecutive cycles present with a mean } \\
\text { relative phase of less than } 30^{\circ} \\
\text { No presence of four or more consecutive cycles with mean } \\
\text { relative phase of more than } 150^{\circ}\end{array}$ \\
\hline Distractor & $\begin{array}{l}\text { Less than } 13 \text { cycles with an absolute mean relative phase of } \\
\text { less than } 30^{\circ} \\
\text { Four or more consecutive cycles present with a mean } \\
\text { relative phase of more than } 150^{\circ}\end{array}$ \\
\hline Problematic & $\begin{array}{l}\text { Less than } 13 \text { cycles with an absolute mean relative phase of } \\
\text { less than } 30^{\circ} \\
\text { No presence of four or more consecutive cycles with a mean } \\
\text { relative phase less than } 30^{\circ} \\
\text { No presence of four or more consecutive cycles with a mean } \\
\text { relative phase more than } 150^{\circ}\end{array}$ \\
\hline
\end{tabular}

the arm movement and the visual signal and they range from $-180^{\circ}$ to $180^{\circ}$ (a negative value indicating that the arm was lagging behind the signal). A lower absolute mean relative phase expresses higher synchronisation accuracy. Less mean within-participant variability corresponds with a more consistent synchronisation performance. Both accuracy and consistency have to be considered to evaluate task performance. Synchronisation with a consistent amplitude and relative phase can be incorrect, for instance, when participants are tracking the distractor surface. Also, absolute mean relative phase can be relatively low, while within-participant variability of relative phase is high indicating that the participant has difficulties staying on target.

All of the above dependent variables were computed as the average of the three trials that each participant completed per combination of coherence level and colour condition. Statistical analyses were performed in a $4 \times 2$ (coherence level $\times$ colour condition) factorial design with repeated measures on both factors.

\subsubsection{Trial classification}

As a complement to the above global parameters, we examined every trial in more detail by labeling each of its back-and-forth cycles according to the absolute mean relative phase for this cycle $(x)$. Each cycle was assigned to one of three groups: indicative of good agreement between the arm movement and the target motion $\left(x<30^{\circ}\right)$, good agreement between the arm movement and the distractor motion $\left(x>150^{\circ}\right)$ or neither $\left(30^{\circ} \leq x \leq 150^{\circ}\right)$. These margins were based on examination of movement patterns in the condition in which performance was optimal (colour condition GR, coherence level 100\%). This labeling enabled us to classify the different trials based on the types of cycles they were composed of. Four mutually exclusive and exhaustive categories of trials were distinguished (see Table 1 for the selection criteria per category): (1) trials dominated by stable synchronisation with the target (Target-trials); (2) trials in which both stable synchronisation with the target and obvious synchronisation problems took place, but in which there was never stable synchronisation with the distractor surface
(TargetDeviant-trials); (3) trials in which there was stable synchronisation with the distractor surface at some point (Distractor-trials), even when there was also good synchronisation with the target during a number of cycles; (4) trials in which synchronisation behaviour was problematic since there was no stable synchronisation with the target, nor with the distractor (Problematic-trials).

\section{Results}

\subsection{Spatial parameters}

Data concerning the spatial parameters are available for inspection in Table 2. First, the mean amplitude was considered. There was a small, yet significant effect of coherence, $F(3$, $45)=4.33, p=.0091$. On average, participants overshot the target amplitude by $8^{\circ}$. There was no significant main effect of colour $(p=.1774)$, nor did we observe a significant interaction effect $(p=.1031)$.

Next, we investigated the mean within-participant variability of amplitude. This was on average significantly higher in the WW condition than in the GR condition, $F(1,15)=20.05$, $p=.0004$. Varying coherence level also had a significant effect, $F(3,45)=3.01, p=.0398$. Both main effects have to be qualified, however, in the light of a significant interaction between the coherence and the colour manipulation, $F(3,45)=10.43$, $p<.0001$. In the WW condition, participants became spatially more consistent in their synchronising movement as coherence decreased. In the GR condition, synchronisation initially became less consistent when the coherence level was lowered, but consistency increased again when coherence reached $10 \%$. It can been seen in Table 2 that in the high coherence conditions (50\% and 100\%) amplitude was less variable in the GR condition than in the WW condition. In the low coherence conditions (10\% and 30\%), however, the reverse situation is observed. In this case amplitude is less consistent in the GR condition.

Additional data concerning amplitude errors yield further insight into the amplitude overshoot. On average participants moved too much to the left (mean: $16.47^{\circ}, \mathrm{SD}: 7.30^{\circ}$ ) and not far enough to the right (mean: $-6.31^{\circ}, \mathrm{SD}: 6.53^{\circ}$ ). This may be due to the fact that the extension movement to the right required more effort than the flexion movement to the left.

\subsection{Spatio-temporal parameters}

The absolute mean relative phase was significantly affected by the colour manipulation, $F(1,15)=8.79, p=.0096$. The spatio-temporal deviation of the arm position from the signal position was on average significantly smaller when target and

Table 2

Means of the spatial synchronisation measurements

\begin{tabular}{|c|c|c|c|c|c|c|c|c|}
\hline & \multicolumn{2}{|l|}{$10 \%$} & \multicolumn{2}{|l|}{$30 \%$} & \multicolumn{2}{|l|}{$50 \%$} & \multicolumn{2}{|l|}{$100 \%$} \\
\hline & WW & GR & WW & GR & WW & GR & WW & GR \\
\hline Amplitude $\left(^{\circ}\right)$ & 55 & 53 & 51 & 51 & 51 & 52 & 51 & 53 \\
\hline Within-participant variability of amplitude $\left(^{\circ}\right)$ & 6 & 7 & 7 & 9 & 8 & 6 & 10 & 5 \\
\hline Amplitude error, left $\left(^{\circ}\right)$ & 16.9 & 16.5 & 16.9 & 16.2 & 15.7 & 16.4 & 16.7 & 16.5 \\
\hline Amplitude error, right $\left(^{\circ}\right)$ & -4.9 & -6.0 & -7.8 & -6.4 & -5.4 & -6.5 & -7.1 & -6.3 \\
\hline
\end{tabular}




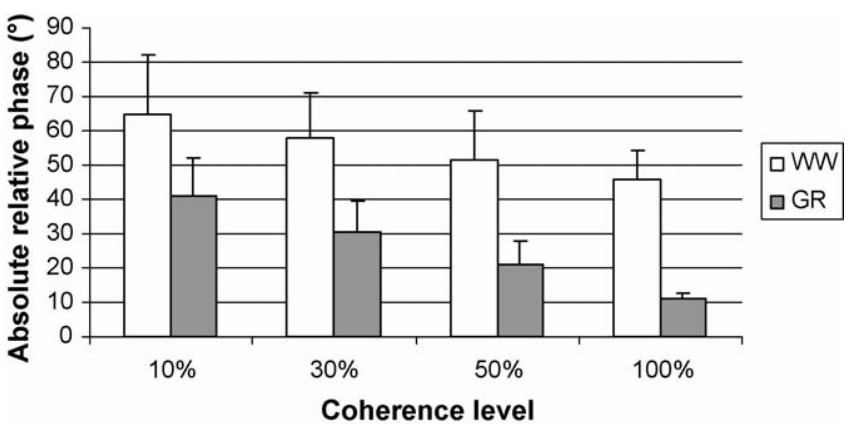

Fig. 1. Mean and standard error of the absolute relative phase $\left({ }^{\circ}\right)$.

distractor surface were coloured differently. The absolute mean relative phase tended to increase as coherence was lowered but this effect was only marginally significant $(p=.0522)$. As can be seen in Fig. 1, this trend was the same for both colour conditions, consistent with a lack of an interaction effect between the colour and coherence manipulations $(p=.9052)$.

Our analysis revealed that implementing a colour difference significantly lowered the mean within-participant variability of the relative phase, $F(1,15)=5.80, p=.0293$, while varying the coherence level yielded no significant effect ( $p=.7872$ ). However, there was a strong interaction between the two main variables, $F(3,45)=17.61, p<.0001$. In Fig. 2, it is apparent that in the GR condition synchronisation performance became less consistent by lowering the coherence level, whereas the opposite occurred in the WW condition. Comparing the colour conditions per coherence level shows that in the high coherence conditions there was less within-participant variability of the relative phase when the target and distractor dots were distinguished by colour, yet, the reverse was true in the low coherence conditions.

\subsection{Trial classification}

In order to understand the results of the analyses on the global spatial and spatio-temporal data, a more fine-grained examination of what goes on in a trial was required. As described in Section 2, we classified each trial in terms of the degree of relatively stable synchronisation with the target or the distractor surface across its back-and-forth cycles (see Table 1 again).

Fig. 3 shows the distribution of the trials per colour and coherence condition ( $n=48,3$ trials for each of the 16 participants)

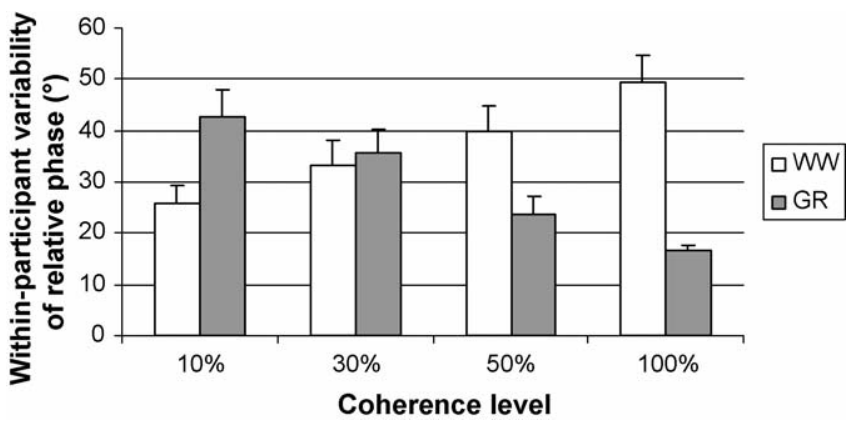

Fig. 2. Mean and standard error of the within-participant variability of relative phase $\left(^{\circ}\right)$.

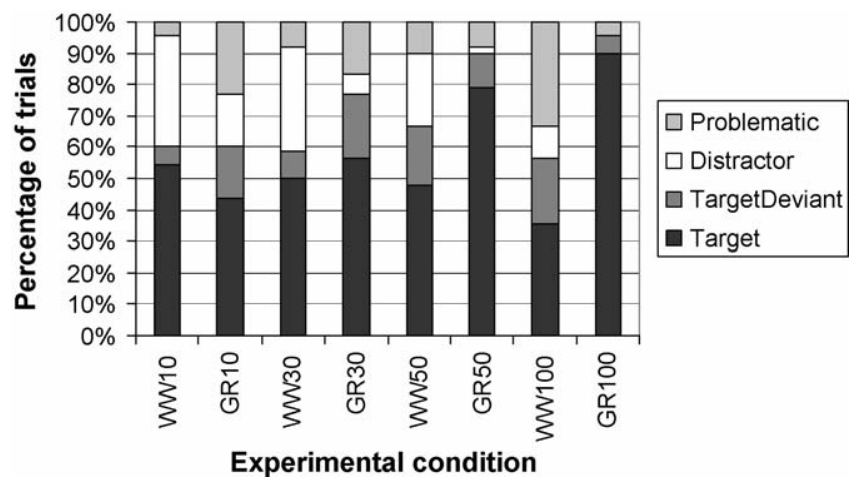

Fig. 3. Distribution of the different trial categories per colour and coherence condition (note: $100 \%=48$ trials, 3 trials for each of the 16 participants).

across the four categories that we distinguished. Let us first take a look at the GR conditions. Participants performed best when target and distractor were coloured differently and were equally salient (i.e., GR 100 condition): $90 \%$ of the trials predominantly consisted of arm movements that corresponded relatively well with the target motion. Decreasing the coherence level, when there was a colour cue present, had an overall negative effect, as we had expected: we observed gradually less Target-trials and gradually more TargetDeviant, Distractor- and Problematictrials. The pattern of results at this finer level matches the results at the more global level: namely, less spatio-temporal accuracy and less consistency with decreasing coherence level.

The results were more complex in the WW condition. Performance was not nearly as good as in the GR condition when both surfaces were equally salient (i.e., the WW100 condition). The relatively low percentage of Target-trials (35\%) and Distractortrials $(10 \%)$ suggests that, in general, participants had difficulty selecting and maintaining attention on either of the two available surfaces. Reducing coherence had both a negative effect (as we expected) and a positive effect, albeit relatively smaller. It resulted in gradually more trials in which the distractor surface was incorrectly selected for tracking at some point (i.e., more Distractor-trials), often early in the trial and without correction. Aside from this negative effect, however, reducing coherence also led to more trials in which stable and accurate synchronisation with the target was obtained. This is indicated by the more moderate increase of Target-trials as the coherence level went down, with the main increase situated between coherence levels $100 \%$ and $50 \%$. TargetDeviant and Problematic-trials became less frequent as the coherence level was lowered. This pattern of results corresponds with the lower average spatio-temporal accuracy, but higher consistency when the coherence level was lowered in the WW condition.

Comparing both colour conditions across coherence conditions, we observed that the number of Distractor-trials was consistently lower in the GR condition than in the WW condition, as expected. Differences for the other trial categories depended on the coherence level. At the highest coherence levels $(50 \%$ and $100 \%)$, Target-trials were clearly more frequent in the GR condition than in the WW condition. At the lower coherence levels (10\% and 30\%), however, there was no substan- 
tial difference in the occurrence of Target-trials; synchronisation difficulties were observed frequently both in the GR and WW condition. There was a difference, however, in how these difficulties manifested. Trials containing stable synchronisation with the distractor occurred more often in the WW condition than in the GR condition. Problematic- and TargetDeviant-trials in which participants were not able to achieve or maintain stable synchronisation with the target, while not locking on to the distractor surface, were more frequent in the GR than in the WW condition. Together, this evidence suggests that a colour cue helped to avoid the distractor surface and, furthermore, aided selection of the target, provided that a stable percept was formed. The colour cue could not compensate for the low signal strength of the synchronisation target at low coherence levels.

\section{Discussion}

In the current study we introduced transparent motion to the action domain. Similar to the study of Ceux et al. [6], participants had to track a complex moving target with their right forearm. The target was a surface, which had to be successfully extracted from the display, moving transparently back-and-forth across another moving surface (i.e., the distractor surface). Following Ceux et al., we manipulated signal strength of the target by making a percentage of dots, which originally constituted the target surface, move randomly. In addition, we introduced a colour cue that distinguished the target from the distractor surface.

We anticipated that when the salience of the synchronisation target was reduced, by lowering the coherence level, synchronisation performance would deteriorate. In addition, we predicted that adding a colour cue would aid proper selection and maintenance of focus on the target and thereby improve synchronisation with the target.

We performed analyses at a global level on the classic measures of synchronisation accuracy and consistency. Consistent with our predictions, we observed that adding the colour cue increased the spatio-temporal accuracy of the synchronisation, as expressed by the absolute mean relative phase. In addition, as we expected, this measure of accuracy decreased by lowering the coherence level, although the effect was only marginally significant. The effect of lowering the coherence level on spatiotemporal consistency of the tracking depended on the presence of a colour cue and vice versa. When a colour cue was present, lowering the coherence level resulted in a decrease in spatiotemporal consistency, while it led to more consistency in the absence of a colour cue. The data on the variability of the subjects' movement amplitude showed a similar interaction.

To elucidate the above results, we complemented our analyses with a detailed examination of each individual trial. This allowed us to classify each trial based on the degree of relatively good synchronisation with the target and the distractor and consider the distribution of the trials across the different categories for each combination of a colour and a coherence condition.

Trial classification showed that performance was optimal when the colour cue was combined with maximal coherence (i.e., the GR100 condition). Furthermore, at all coherence lev- els, adding the colour cue reduced the amount of synchronisation attempts in which stable synchronisation with the distractor surface took place. When the coherence level was lowered, in the presence of a colour cue, synchronisation difficulties ensued, as expected. Trial classification further revealed why our mean measure of spatio-temporal consistency increased as coherence was reduced in the WW condition. When a colour cue was absent, synchronisation difficulties, unexpectedly, occurred at all coherence levels. The expression of these difficulties differed across coherence levels. When coherence was maximal, participants rarely settled into stable synchronisation with the distractor surface in case of problems. As coherence was reduced, however, there were more trials in which participants began tracking the distractor surface smoothly but incorrectly. This explains the increased consistency. Finally, trial classification brought a difference into view between the frequent synchronisation problems at low coherence levels in the condition with a colour cue and in the condition without a colour cue. When a colour cue was absent, there were relatively more trials in which the distractor surface was well synchronised with. When a colour cue was present, there were more trials in which participants had difficulty to achieve or maintain stable synchronisation with the target but did not go into stable synchronisation with the distractor surface.

This complex pattern of results becomes less surprising when one considers that multiple processes are involved in our synchronisation task (see Fig. 4) and that our manipulations are likely to have affected several of them. We propose the following framework. First, participants have to properly segment the display once the transparent motion stimulus appears. If segmentation is achieved, the target surface can be selected as the one whose motion direction logically follows that of the 10 preceding dots (in the WW condition) or on the basis of this prior information and the colour cue (in the GR condition). When the target is selected, the participant has to maintain focus on it, while a potentially distracting surface moves in the opposite direction. This process can be aided by the colour cue. When the coherence level is low, segmentation may be (initially) unsuccessful or result in an unstable target surface. In the former case, target selection will be impossible. In the latter case, the target may be selected but the process of maintaining focus is likely to fail occasionally. Either way, attention will be attracted by the salient distractor surface which is perfectly coherent. If the distractor is selected for tracking, error detection and correction

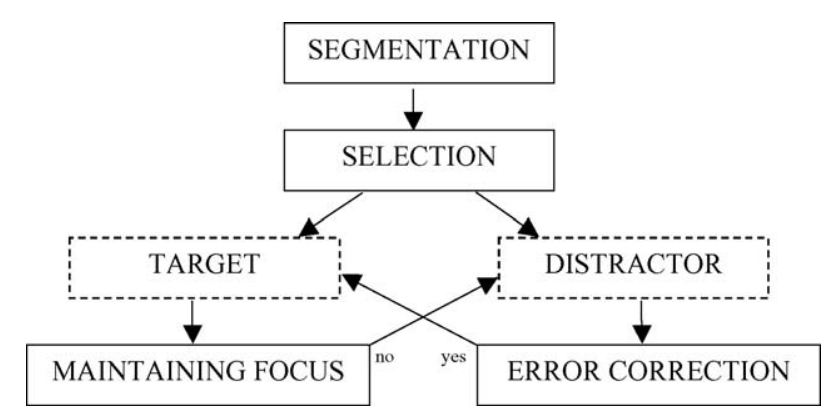

Fig. 4. Proposed framework of processes involved in synchronisation task. 
can ensue based on the colour cue, although not always successfully. Indeed our results indicate that the colour cue acted as a top-down cue that could be used to avoid incorrect tracking of the distractor surface, through promotion of corrective behaviour. It also ensured stable tracking of the target, but only when the bottom-up signals underlying the target were sufficiently strong, that is, at high coherence levels when a salient target surface percept was achieved.

Further research should attempt to isolate the different contributions of our manipulations to the synchronisation task in order to verify our framework. For instance, in our task the colour cue served as an attention cue by indicating what was relevant, but it was also the only cue that provided information during the trial about what the target was. This means that if segmentation was initially unsuccessful, or the target was lost during the trial, it could still be found. To investigate the contribution of attention in isolation, the angle between the two motion directions in the stimulus could be altered, so that target identity remains available throughout the trial in both colour conditions via motion direction information. Another suggestion for future research is to combine our synchronisation task with eye movement tracking. This could provide additional information about some unexpected results and about the inter-individual variability in the synchronisation task. Indeed, we unexpectedly observed more trials dominated by good synchronisation with the target as coherence level was lowered in the WW condition, and some participants did well in virtually all conditions. This could reflect the use of a strategy; accurate synchronisation may have been achieved by moving the right arm oppositely to the distractor surface. It has been shown that participants are able to synchronise in opposite direction of a coherent plane, although their synchronisation behaviour is more variable compared to in-phase synchronisation [6]. Eye movement patterns could be indicative of such a strategy. Finally, it would be interesting to study participants' adaptive behaviour in the current task in future experiments. This could be done by breaking the rhythmicity of the stimulus motion through a frequency change during the trial, as Ceux et al. [7] did in coherent motion stimuli. Another possibility would be to start all trials with a colour cue available, but at a fixed time either keep or drop the colour information. This would ensure that the target could be properly identified at the start of the trial when segmentation was successful.

We hope that our findings, the framework to understand them, and the suggested research questions that can test it, will inspire further investigation of motor performance coupled to complex motion stimuli, and in particular of the coupling between synchronisation and transparent motion.

\section{Acknowledgements}

This research was supported by a research grant from the Fund for Scientific Research - Flanders (G.0095.03) and by the Concerted Research Effort Convention of the Research Fund K.U. Leuven (GOA/2005/03-TBA). We thank Frans A.J. Verstraten for providing us the initial Motion Coherence Matlab code which we adapted. We thank Pedro Rosas for aiding us with this adaptation. We are also thankful for the valuable comments of our reviewers.

\section{References}

[1] Blaser E, Pylyshyn ZW, Holcombe AO. Tracking an object through feature space. Nature 2000;408:196-9.

[2] Brainard DH. The Psychophysics Toolbox. Spat Vis 1997;10:433-6.

[3] Britten KH, Shadlen MN, Newsome WT, Movshon JA. The analysis of visual motion: a comparison of neuronal and psychophysical performance. J Neurosci 1992;12:4745-65.

[4] Buekers MJ, Bogaerts HP, Swinnen SP, Helsen WF. The synchronization of human arm movements to external events. Neurosci Lett 2000;27:171-4.

[5] Ceux T, Buekers MJ, Montagne G. The effects of enhanced visual feedback on human synchronization. Neurosci Lett 2003;290:181-4.

[6] Ceux T, Wagemans J, Rosas P, Montagne G, Buekers MJ. The influence of motion coherence manipulations on the synchronization level of a perception-action task. Behav Brain Res 2005;162:83-9.

[7] Ceux T, Wagemans J, Rosas P, Montagne G, Buekers MJ. Perceptual-motor adaptations in a synchronization task: the joint effects of frequency and motion coherence manipulations. Behav Brain Res 2006;168:226-35.

[8] Croner LJ, Albright TD. Image segmentation enhances discrimination of motion in visual noise. Vision Res 1997;37:1415-27.

[9] Pelli DG. The VideoToolbox software for visual psychophysics: transforming numbers into movies. Spat Vis 1997;10:437-42.

[10] Richmond Eye Associates: vision tests (n.d.). Colour vision test. Retrieved on November 2003 from http://www.richmondeye.com/ colourframe.htm.

[11] Scholl BJ. Objects and attention: the state of the art. Cognition 2001; 80:1-46.

[12] Sekuler R, Watamaniuk SNJ, Blake R. Perception of visual motion. In: Yantis S, Pashler H, editors. Stevens' handbook of experimental psychology. Sensation and perception, vol. 1, 3rd ed. New York: John Wiley \& Sons Inc.; 2002. p. 121-76.

[13] Snowden RJ, Verstraten FAJ. Motion transparency: making models of motion perception transparent. Trends Cogn Sci 1999;10:369-77.

[14] Uttal WR, Spillmann L, Sturzel F, Sekuler AB. Motion and shape in common fate. Vision Res 2000;40:301-10.

[15] Valdes-Sosa M, Cobo A, Pinilla T. Transparent motion and object-based attention. Cognition 1998;66:B13-23.

[16] Watamaniuk SNJ, Sekuler R, Williams DW. Direction perception in complex dynamic displays: the integration of direction information. Vision Res 1989;29:47-59.

[17] Wertheimer M. Gestalt theory. In: Ellis D, editor and translator. A source book of Gestalt psychology. New York: Harcourt; 1938. p. 1-11. 